\title{
SURFACE FORCES IN CHEMICAL MECHANICAL PLANARIZATION AND SEMICONDUCTOR WAFER CLEANING SYSTEMS
}

\section{Lukasz Hupka ${ }^{1}$, Jakub Nalaskowski ${ }^{2}$, Jan D. Miller ${ }^{3}$ and Jan Hupka ${ }^{1}$}

\author{
${ }^{1}$ Gdansk University of Technology \\ ${ }^{2} S U N Y$ Polytechnic Institute \\ ${ }^{3}$ University of Utah \\ *e-mail:lukasz.hupka@pg.edu.pl
}




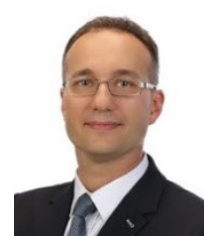

Lukasz J. Hupka earned B.S. degree in Environmental Protection and Management from the Gdansk University of Technology in 2002, M.S. and $\mathrm{PhD}$ in Metallurgical Engineering from the University of Utah in 2006 and 2008 respectively. In 2008-2010 he was a post-doc at IBM Thomas J. Watson Research Center. In 2010 - 2016 Hupka worked in R\&D process development at Micron Technology, Inc. Since 2017 Lukasz Hupka is leading projects concerning surface chemistry, energy storage, and lithium-ion batteries recycling.

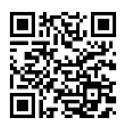

(iD)

https://orcid.org/0000-0003-1616-1944

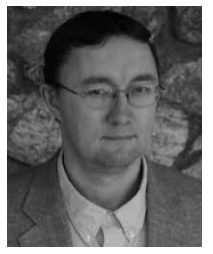

Jakub Nalaskowski received Ph.D. in Chemical Technology from Gdansk University of Technology, in 1999. He served as Research Associate Professor at the University of Utah. Since 2008 he worked at IBM T. J. Watson Research Center, Cabot Microelectronics, and SEMATECH in scientific and technical leadership roles related to CMP process, slurry, and pad development. In 2016 he joined SUNY Polytechnic Institute as Planarization Center Technical Leader at Albany Nanotech Complex. His areas of expertise include fundamental and applied aspects of CMP, wet surface chemistry, interfacial forces measurement, and scanning probe microscopy.

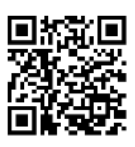

https://orcid.org/0000-0002-5819-750X

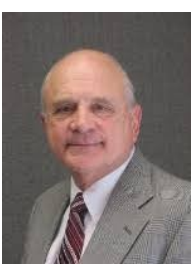

Jan D. Miller is the Ivor D. Thomas Distinguished Professor of Metallurgical Engineering in the College of Mines and Earth Sciences at the University of Utah. He was elected to the National Academy of Engineering in 1993. His research covers mainly the areas of mineral processing and hydrometallurgy, specializing in particle characterization, aqueous solution chemistry, and colloid and surface chemistry.

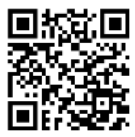

iD

https://orcid.org/0000-0003-4889-1108

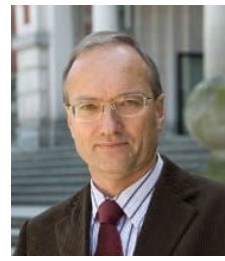

Jan Hupka is employed in the Department of Process Engineering and Chemical Technology, Chemical Faculty at the Gdansk University of Technology. Jan Hupka has been also associated with the University of Utah. Professor Hupka major research interest is in applied surface chemistry as used in waste treatment, TiO2-based photocatalysis, mineral processing, process engineering and environment protection against pollution.

https://orcid.org/0000-0002-9713-6902 


\begin{abstract}
Superior uniformity and local planarity of semiconductor wafers in the chemical mechanical planarization (CMP) process as well as efficient post-CMP cleaning is controlled by surface chemistry phenomena. The AFM colloidal probe technique was used to demonstrate surface forces which are of special significance to CMP and post-CMP cleaning. Examples of ways to manipulate those interactions are provided, and the benefits to CMP processes and post-CMP cleaning are discussed.
\end{abstract}




\section{INTRODUCTION}

Chemical mechanical planarization (CMP) is an important semiconductor process enabling integration of emerging semiconductor devices and continual decrease in physical dimensions of memory and microprocessor chips. As implied by the name; CMP incorporates removal of materials by a unique combination of chemical and abrasive action to achieve highly planar surfaces that are also very smooth [1]. CMP process fundamentals as well as applied aspects and consumables are provided in detail in the literature [2].

Whether the abrasive particle (CMP process) or contaminant particle (cleaning process) will come close to the surface or perhaps even adhere to the surface, depends on the net force acting between the particle and the substrate. The net force is assembled from constituent forces such as electrostatic force and van der Waals (included in Derjaguin Landau, Verwey and Overbeek -DLVO theory [3]) and nonDLVO forces such as solvation, hydration, steric or hydrophobic forces. Depending on the system and conditions one of the forces can dominate over all others, as shown in Figure 1 [4-5].

The forces are either attractive or repulsive. In this system hydrophobic interactions and van der Waals forces are attractive, and the electrical double layer, steric and hydration forces are repulsive. As shown in Figure 1 it is possible that the particle will be repelled at some distance from the surface, and once the energy barrier is overcome, attraction may occur, and the particle will eventually attach.

In the case of CMP and post-CMP cleaning systems usually electrical double layer (EDL) interactions and steric forces are most significant. Electrical double layer, because almost any surface is charged in aqueous medium and steric force, because of the unique compositions of CMP slurries.

\section{MEASUREMENT TECHNIQUE}

Atomic Force Microscopy (AFM) is a versatile tool capable of measuring surface topography, friction, magnetic and capacitance properties. AFM can also be used to measure interaction and adhesion forces between the cantilever tip or a particle attached to the cantilever and nearly any surface.

The AFM cantilever during force measurement acts as a spring. A photodetector records the deflection of the cantilever as a function of its distance from the surface. With a known cantilever constant $k$ (spring constant) and deflection (displacement) the interaction force is calculated according to Hooke's law. The AFM colloidal probe technique is a reliable method for interaction force measurement in aqueous medium [6-7]. Force measurement can be done with the 
cantilever tip or with a particle glued to a tipless cantilever such as the $20 \mu \mathrm{m}$ aluminum oxide particle (Figure 2A) or the $0.7 \mu \mathrm{m}$ silica particle (Figure 2B) [8].

\section{SURFACTANTS AND SURFACE FORCES}

Removal rate during CMP as well as the efficiency of post-CMP cleaning is controlled by chemical and mechanical components of the processes. Electrical Double Layer (EDL) and Steric Forces are dominant interactions observed in CMP and post-CMP cleaning systems.

Surfactants are commonly used in CMP and post-CMP cleaning. Their role is indispensable.

Many materials and films currently used in the semiconductor industry are hydrophobic (silicon, silicon nitride, poly) as well as many consumables (i.e. CMP polishing pads). Hydrophobic interactions are one of the strongest, yet are understood and utilized very little.

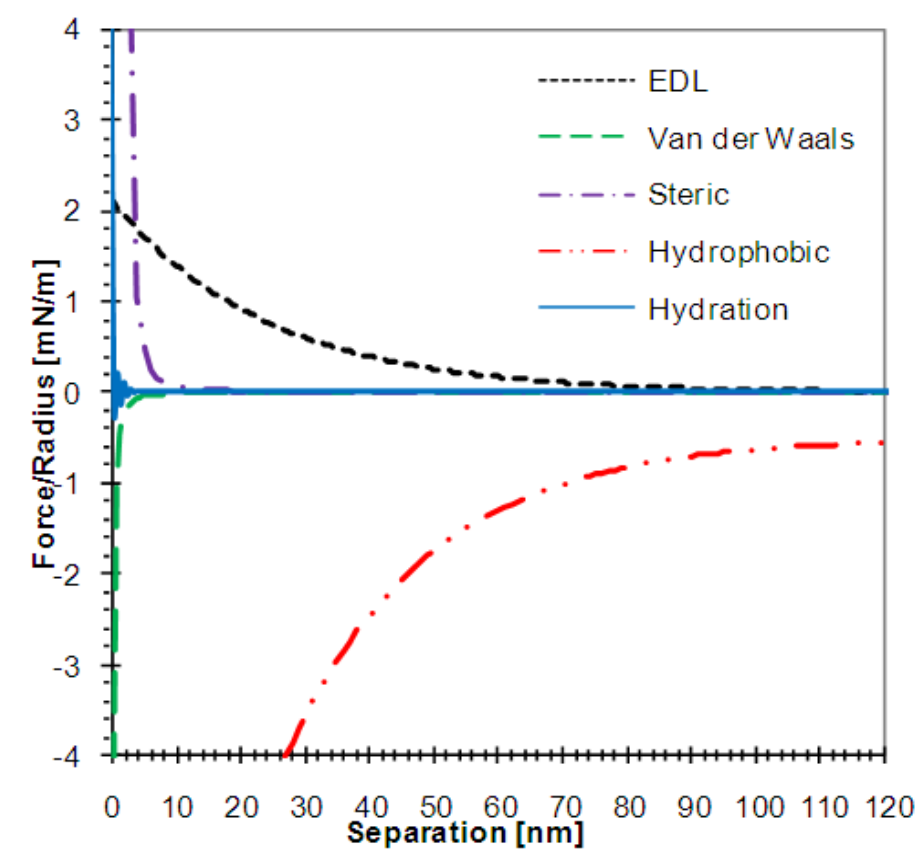

Figure 1. Interaction forces in an aqueous environment; comparison of their magnitude and acting distance 
A

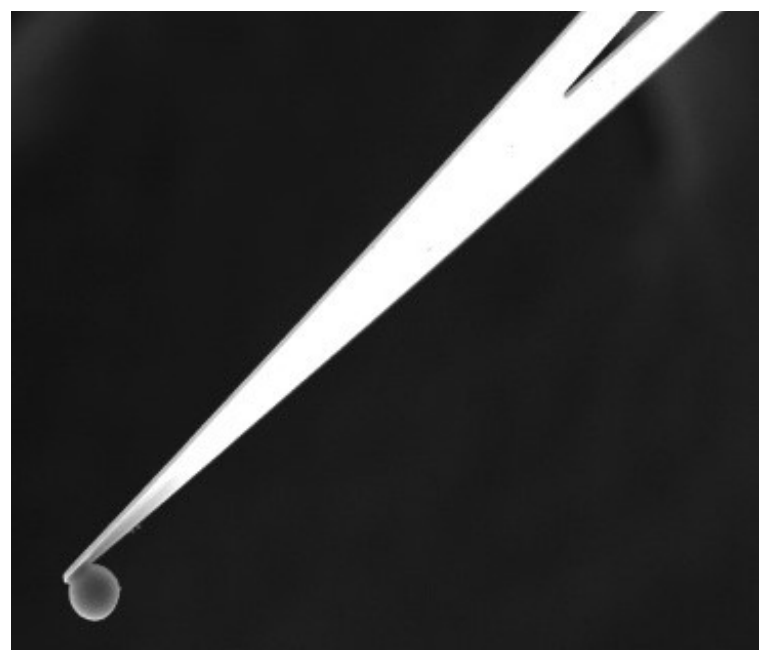

B

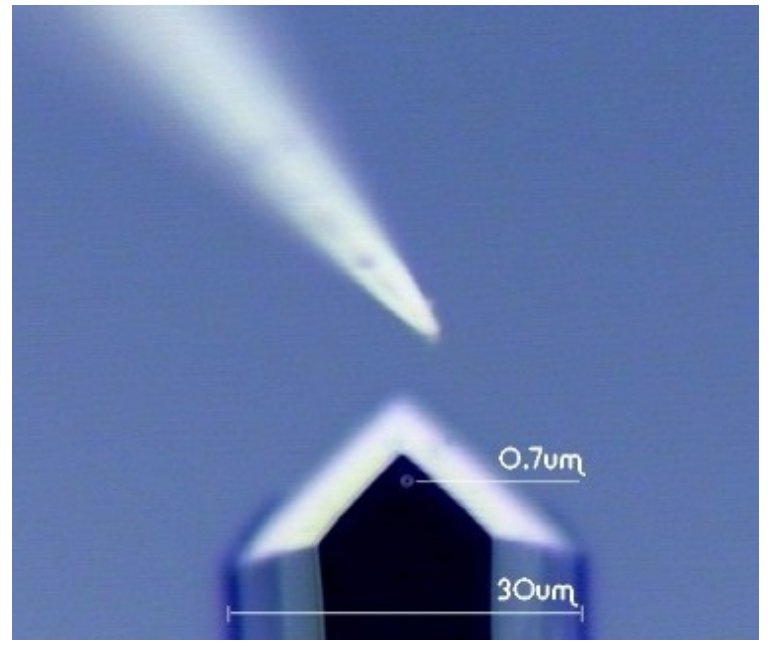

Figure 2. AFM colloidal probes for measurement of interaction forces, as seen under SEM and optical microscopes. A. Cantilever tip with glued $20 \mu \mathrm{m}$ aluminum oxide particle. B. Cantilever tip with glued very fine silica particle

Surfactants play a central role in CMP and post-CMP cleaning. Some of the surfactants' significance in semiconductor wafer planarization includes:

1). Material selectivity tuning and stop-on capability enablement - selectivity of the process is increased thanks to surfactant adsorption and passivation of selected materials. For example, slurry is designed for selectivity close to $1: 1$, but when surfactants are added silicon oxide to silicon nitride selectivity is increased to 
20:1 or even higher. Silicon oxide to polysilicon selectivity can be as high as 500:1 [9].

2) Rate of removal alteration; increase or decrease of material removal depending on application. Thick layers of oxide are used in new vertical integration schemes (etching into the $\mathrm{Si}$ wafer, rather than building layers) - these are characterized by a large step height (microns) that needs to be planarized. High oxide rate slurries are used and their removal rate is increased by surface active additives [10-11].

3) Defect reduction - surfactants are used to decrease defects during planarization process; reduce scratches, minimize slurry particle adhesion, minimize particle contamination form material which is being polished, etc.) [1213].

4) Endpoint detection - surfactants are used to tune endpoint signal by altering friction between platen and wafer. Surfactants can be beneficial in planarity detection and overburden reduction [14].

5) Planarization efficiency improvement. Surface active ingredients promote removal of topography and protect "downs" from dishing [15].

6) Surfactants improve tribology, improve process lubrication, pad wettability and slurry distribution between wafer and polishing pad. Proper slurry transport between polishing pad and silicon wafer contribute greatly to uniformity of the CMP process [16].

7) Post-CMP cleaning - surfactants are used in post-CMP cleaning and are effective in particle adhesion prevention, removal and suspension in cleaning solution. Surfactants can be used during spraying operation, in polyvinyl alcohol (PVA) brushes cleaning solution, in megasonic tanks, and cross-contamination prevention stations [17-19].

Surfactants can affect and help control interaction forces in CMP and postCMP cleaning. Thanks to their properties and structure, surfactants can influence electrical double layer, steric force and hydrophobic interactions.

\section{A. Electrical Double Layer (EDL)}

Electrical Double Layer (EDL) force related to the presence of electric charge and double layer of ions at the interface at the interface solid and liquid phase. EDL forces strongly depend on surface and solution chemistry. EDL forces can be modified by changing solution chemistry (salt concentration, $\mathrm{pH}$, adsorbing ions). EDL forces can be attractive or repulsive depending on the surface charge as shown on Figure 3 [19]. 


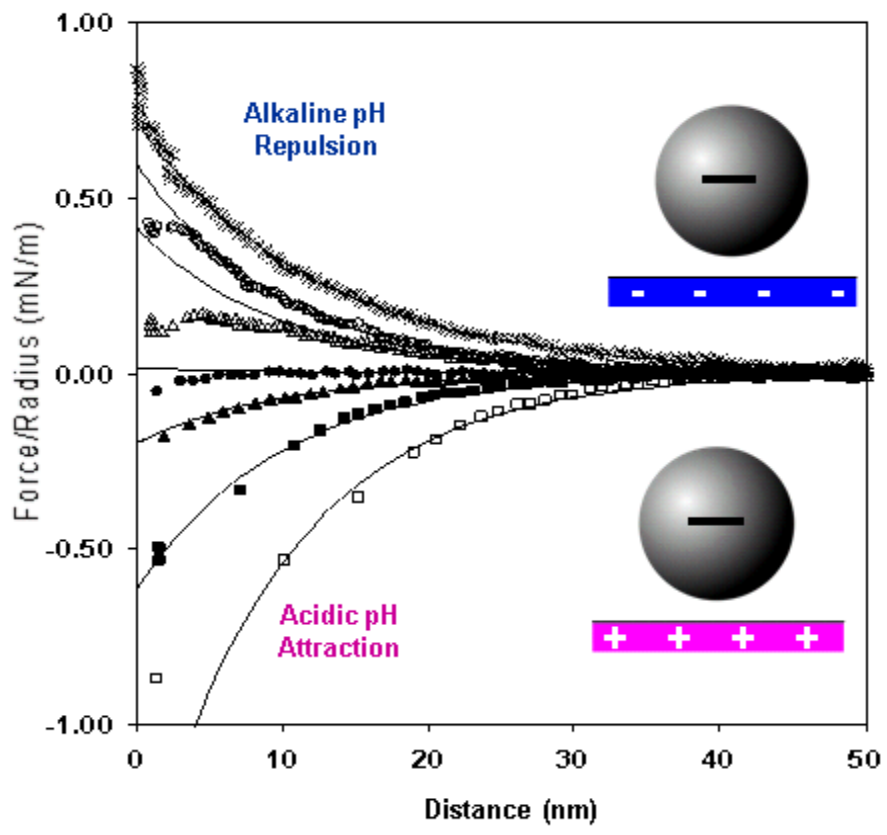

Figure 3. Force versus separation distance for interactions between silica spherical probe and a fluorite optical window in $1 \times 10^{-3} \mathrm{M} \mathrm{NaNO}_{3}$ as a function of $\mathrm{pH}$. The symbols show the AFM results, and the line shows the theoretical curve

By changing $\mathrm{pH}$ of CMP slurry/post-CMP cleaning solution the interaction forces between particle and wafer surface can be manipulated from highly attractive to highly repulsive [4], [20].

\section{B. Steric Forces}

A molecular film on the wafer and abrasive surface can affect both chemical and mechanical components of the CMP process. The molecular film is a diffusion barrier for chemical component action (hydration, hydrolysis, etching) and at the same time force barrier for slurry particle - wafer surface mechanical interaction. There are a number of surface active film forming additives which are currently being used or are still under development to provide superior selectivity, planarity and uniformity. Most of them can be classified as surfactants (anionic, cationic, nonionic), polymers (uncharged, charged - polyelectrolytes) or amino acids. Example of how interaction forces can be modified by formation of molecular film at the surface is shown on Figure 4 [21], [5].

In the case of symmetric aluminum oxide system, a small repulsion was significantly enhanced by introduction of a surfactant. Surfactant micelles adsorb 
on the alumina surface forming a protective steric layer that can be beneficial in providing high selectivity during CMP process or can provide repulsive barrier during post-CMP cleaning.

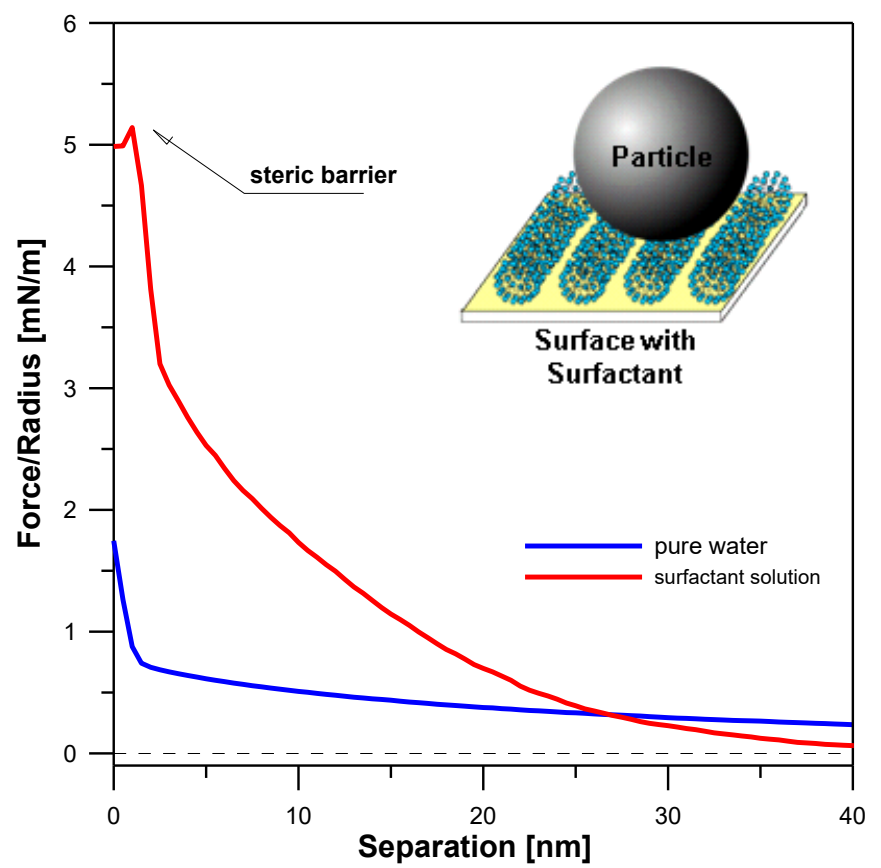

Figure 4. Repulsive forces between alumina particle and alumina surface in deionized (DI Milli-Q) water and in a surfactant system. Surfactant adsorbs on the surface forming protective steric barrier

\section{Hydrophobic Interactions}

Hydrophobic interactions belong to the strongest forces observed at the nanoscale, yet they seem to be the least understood interactions, currently described only by empirical equations. They are characterized by a strong attraction between hydrophobic surfaces in water, often accompanied by formation of bridging cavities [6] and nanobubbles, [7], [22] see Figure 5. [23] 


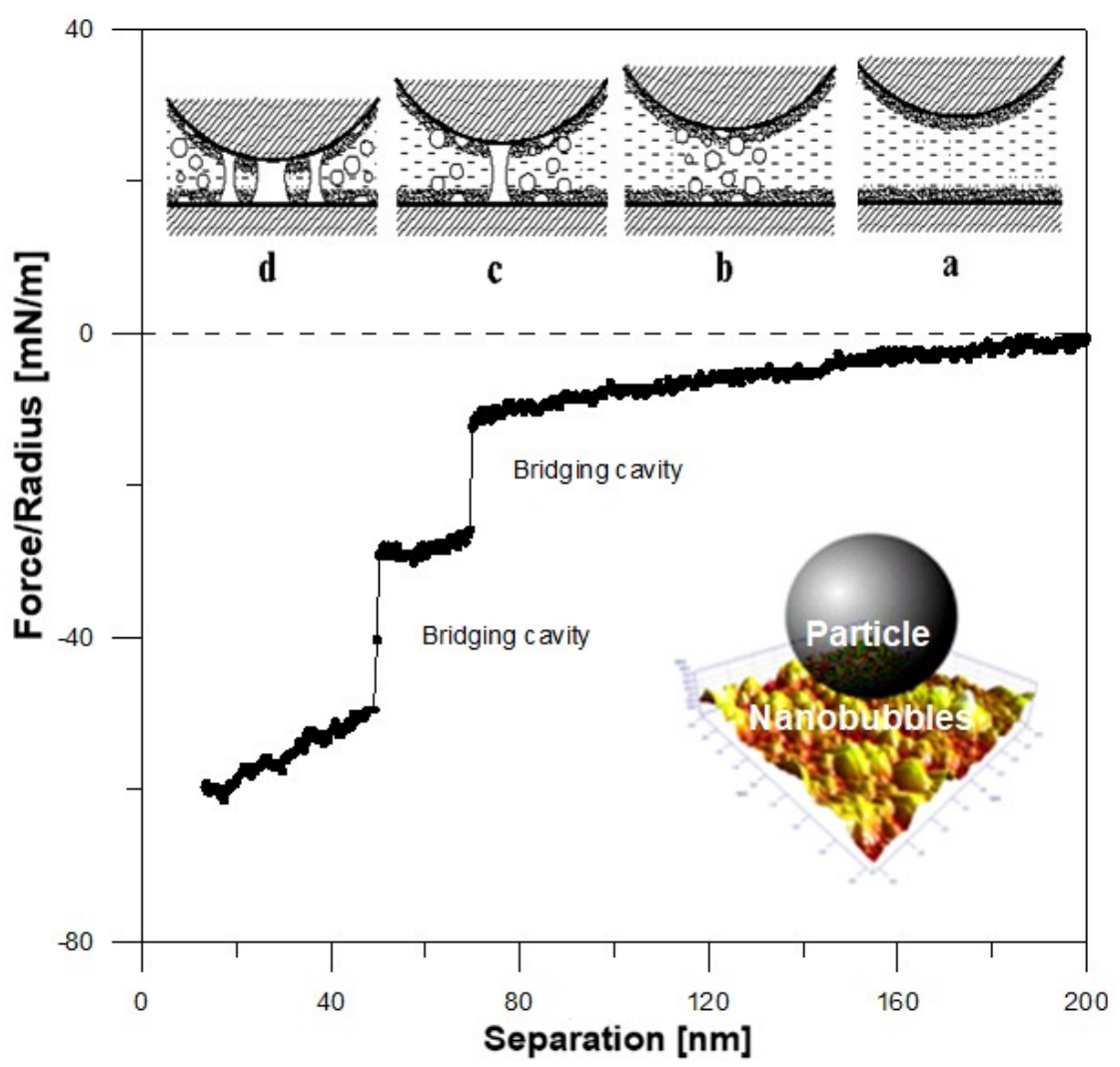

Figure 5. Interaction force curve for silanated silica (106 deg. contact angle). Symmetric system reveals strong hydrophobic attraction escalated by formation of bridging cavities and possible nanobubbles. The hypothetical hydrophobic attraction mechanism is proposed in Figures 5a to 5d. a) Hydrophobic colloidal probe approaches hydrophobic surface, b) At the distance from $180 \mathrm{~nm}$ to $200 \mathrm{~nm}$ local vacuum appear starting enhanced hydrophobic attraction, c) single bridging cavity is formed as indicated by sudden jump in the attractive force at $70 \mathrm{~nm}$ distance, d) multiple bridging cavities are formed as indicated by the next even stronger attractive jump at around $50 \mathrm{~nm}$ distance

\section{CONCLUSIONS}

1. Surfaces composed from different materials interact with the force depending upon the surface charge, surfactant adsorption density and form, and the adhesion/cohesion effect. Electrical double layer interactions usually extend up to 100 $\mathrm{nm}$ in CMP-related systems. Surfactant micelles form firm steric barrier at $3.5-4 \mathrm{~nm}$, and contribute to the repulsive forces extending to $25 \mathrm{~nm}$. One can deduce that at $2 \mathrm{~nm}$ from the investigated surface surfactant structures are broken and particle is attracted into contact. 
2. Systems involving hydrophobic interactions seem to be an area of opportunity for CMP and wet processing.

3. Consumables used in CMP and semiconductor materials being planarized are often hydrophobic. Hydrophobic interactions extend to hundreds of nanometers. AFM investigation using self-prepared $0.7 \mu \mathrm{m}$ spherical probe, simulating CMP slurry particle, revealed that hydrophobic interactions in the model system extended to 200 $\mathrm{nm}$. Bridging cavities occurred at $70 \mathrm{~nm}$ and $50 \mathrm{~nm}$ separation distances.

4. Manipulation and control of hydrophobic interactions, bridging cavities and formation of nanobubbles can enhance CMP, post-CMP cleaning, and the wet process technology. Thus, higher removal rates, selectivity, superior planarity and uniformity are achieved during planarization process. Subsequently, low defects and high yields are achieved in the post-CMP cleaning.

\section{REFERENCES}

[1] M. Krishnan, J. Nalaskowski, and L. Cook, Chem. Rev., 2009, 110, 178.

[2] P.B. Zantye, A. Kumar, A.K. Sikder, Mater. Sci. Eng. R Rep., 2004, 45, 89.

[3] J.N. Israelachvili, Intermolecular and surface forces, Academic Press, London, 1992.

[4] M.R. Uhlig, S. Benaglia, R. Thakkar, J. Comer, R. Garcia, Nanoscale, 2021, 13, 5275.

[5] L. Hupka, Particle-Surface Interactions in Post-Lap Cleaning of Alumina/Titanium Carbide Wafers, University of Utah, Salt Lake City, 2006.

[6] J. Nalaskowski, J. Drelich, J. Hupka, J.D. Miller, Langmuir, 2003, 19, 5311.

[7] B. Babel, M. Rudolph, MethodsX, 2019, 6, 651 .

[8] L. Hupka, J. Nalaskowski, N. Sinha, J. Greeley, Z. Clark, H. Du, W. Johnson, J. Miller, Solid State Phenomena, 2009, 145, 77.

[9] J. Nalaskowski, 13th International Symposium on Chemical-Mechanical Planarization, CAMP Clarkson University, New York, 2010.

[10] Q. Xu, L. Chen, J. Liu, H. Cao, ECS J. Solid State Sci. Technol., 2019, . 8, 370.

[11] Q. Xu, L. Chen, H. Cao, J. Liu, ECS J. Solid State Sci. Technol, 2021, 10, 1149.

[12] Y. Li, Y. Liu, C. Wang, X. Niu, T. Ma, and Y. Xu, ECS J. Solid State Sci. Technol., 2018, 7, 317.

[13] B. Wu, ECS J. Solid State Sci. Technol, 2021, 10, 3.

[14] Q. Xu, F. Yang, L. Chen, H. Cao, Int. J. of Prec. Eng. and Mfg., 2018, 19, 1585.

[15] C. Yang, X. Niu, J. Zhou, J. Wang, Z. Huo, Y. Lu, ECS J. Solid State Sci. Technol., 2020, 9, 534.

[16] C. Wang, Y. Liu, C. Wang, H. Zhang, China Semiconductor Technology International Conference (CSTIC), 2019.

[17] S. Tian, B. Tan, Q. Wang, C. Han, L. Yang, B. Gao, China Semiconductor Technology International Conference (CSTIC), 2019.

[18] Q. Wang Colloids Surf. -Physicochem. Eng. Asp., 2020, 2, 586.

[19] S. Assemi, J. Nalaskowski, J.D. Miller, W.P. Johnson, Langmuir, 2006, 22, 1403.

[20] J. Nalaskowski, 10th International Symposium on Wafer Cleaning and Surface Preparation, Boise, ID, 2006.

[21] Nalaskowski J., Hupka L., Miller J.D., 9th International Symposium "Surface Preparation to the Forefront: Advanced Materials and Technologies for 65nm and Below,” Boise, ID, Jun. 2005. 
[22] J. Hupka, A. Tonderski, R. Aranowski, C. Jungnickel, Oils and Fuels for Sustainable Development, Gdansk, 2008.

[23] L. Hupka, Particle-wafer interactions in semiaqueous silicon cleaning systems, University of Utah, Salt Lake City, 2008.

Praca wpłynęła do Redakcji 5 czerwca $2021 \mathrm{r}$. 